Cahiers $d u$ MONDE RUSSE

\section{Cahiers du monde russe}

Russie - Empire russe - Union soviétique et États indépendants

$49 / 1 \mid 2008$

Réformes et société en Asie centrale tsariste et soviétique

\title{
Hommage à Marc Raeff
}

\section{Alain Besançon}

\section{OpenEdition}

\section{Journals}

Édition électronique

URL : https://journals.openedition.org/monderusse/9113

DOI : 10.4000/monderusse. 9113

ISSN : $1777-5388$

Éditeur

Éditions de l'EHESS

Édition imprimée

Date de publication : 25 février 2008

Pagination : 7-12

ISBN : 978-2-7132-2195-8

ISSN : $1252-6576$

Référence électronique

Alain Besançon, "Hommage à Marc Raeff », Cahiers du monde russe [En ligne], 49/1 | 2008, mis en ligne le 01 janvier 2009, consulté le 03 septembre 2022. URL : http://journals.openedition.org/ monderusse/9113; DOI : https://doi.org/10.4000/monderusse.9113 
chercher : repérer : avancer

Cet article est disponible en ligne à l'adresse :

http://www.cairn.info/article.php?ID REVUE=CMR\&ID NUMPUBLIE=CMR 491\&ID ARTICLE=CMR 4910007

\title{
Hommage à Marc Raeff
}

\author{
par Alain BESANÇON
}

\section{| Editions de l'EHESS | Cahiers du monde russe}

\author{
2008/1 - Vol 49 \\ ISSN 1252-6576 | ISBN 9782713221958 | pages 7 à 12
}

Pour citer cet article :

- Besançon A., Hommage à Marc Raeff, Cahiers du monde russe 2008/ 1, Vol 49, p. 7-12.

Distribution électronique Cairn pour les Editions de l'EHESS.

(C) Editions de l'EHESS. Tous droits réservés pour tous pays.

La reproduction ou représentation de cet article, notamment par photocopie, n'est autorisée que dans les limites des conditions générales d'utilisation du site ou, le cas échéant, des conditions générales de la licence souscrite par votre établissement. Toute autre reproduction ou représentation, en tout ou partie, sous quelque forme et de quelque manière que ce soit, est interdite sauf accord préalable et écrit de l'éditeur, en dehors des cas prévus par la législation en vigueur en France. Il est précisé que son stockage dans une base de données est également interdit. 


\section{HOMMAGE À MARC RAEFF}

Il y a quelques années (en 1991, je crois) un colloque d'histoire russe s'est tenu à l'EHESS. Marc Raeff était présent. En lui donnant la parole, le président le fit en ces termes : «Marc Raeff. No further introduction needed». Il eût été en effet inutile voire indécent de le présenter. Pour tous ceux qui avaient quelque rapport avec la Russie, avec son histoire et sa civilisation, il était le maître par excellence. Nous avons la douleur et le devoir de faire aujourd'hui cette présentation.

Marc Raeff est né à Moscou en 1923. Son père, un ingénieur de tendance libérale, participait assez activement à la vie politique. L'enfant avait l'air fragile et il était handicapé par une malformation du palais. Sa formidable mère fit face à tous les soins et à toutes les opérations réparatrices avec la même énergie qui la conduisit, sans fléchir, au-delà de cent ans.

Participer à la vie intellectuelle et politique, en 1923, signifiait risquer au pire la mort, l'émigration, au mieux. La famille s'installa à Berlin et l'enfant apprit l'allemand à l'école primaire. Le nazisme eut au moins ceci d'heureux que Marc fit ses études secondaires au Lycée Michelet, à Vanves, auquel il garda une fidèle reconnaissance. Il y apprit le français, et aussi bénéficia de la très solide éducation que dispensaient les bons lycées français de ce temps-là. Marc Raeff illustre parfaitement cette vérité qu'on ne peut juger équitablement de la culture russe, et garder à son endroit le sens des proportions, que si on connaît vraiment au moins une autre culture. Il en savait deux de plus, l'allemande et la française auxquelles il demeura toujours attaché, plus encore, me disait-il, qu'à l'anglaise et à l'américaine. Avec la langue, il entrait dans la littérature, la philosophie, l'histoire de ces pays d'accueil dont il approfondit la connaissance jusqu'à la fin de sa vie. Il était un lecteur sage et infatigable, tout le contraire d'un spécialiste, en cela de la race des grands Européens de l'ancien temps. En reste-t-il beaucoup ?

1941 : la famille passa l'océan et Marc s'engagea dans l'armée américaine. Elle sut faire bon usage de sa polyglottie. C'est finalement en anglais, dans un bon anglais clair et ferme que Raeff écrivit la majeure partie de son œuvre - mais il publia aussi en français, et encore en allemand et en russe, avec autant de plaisir, sinon davantage. À la fin de la guerre, il était chargé de communiquer avec les personnes déplacées ou les prisonniers, que des équipes soviétiques prenaient en main et s'efforçaient de faire rentrer au pays où les attendait au minimum le camp de travail. Ils le savaient et le jeune Raeff avait beau les avertir, ils rentraient quand même. 
Démobilisé, Marc Raeff bénéficia du GI bill qui lui donnait la possibilité d'entrer à l'université. Pour lui ce fut Harvard.

Il faut ici mentionner un épisode considérable de la vie intellectuelle. L'Amérique savante ne s'était pas tellement occupée de la Russie. Il existait quelques travaux classiques - par exemple ceux de Geroid Robinson-, mais on peut dire que jusqu'à la Seconde Guerre mondiale, la France, l'Allemagne, l'Angleterre en avaient fourni davantage. Or, pour des raisons évidentes, il était urgent qu'au lendemain de cette guerre, dont la conclusion avait sérieusement souffert de l'ignorance de la nature du régime soviétique, l'Amérique fût informée. De même qu'à partir d'un groupe de savants, le plus souvent récemment immigrés, les États-Unis avaient pu organiser l'immense program de la bombe atomique, de même, en puisant là aussi dans l'immigration, elle fut en mesure d'aboutir à une vue historique et politique cohérente, suffisamment complète de la Russie soviétique dans ses principaux aspects. Le Harvard Russian Center fut au centre de ce program d'un autre type. Si divers que fussent les auteurs, ils convergeaient et la version qu'ils mirent à la disposition du monde savant comme des responsables politiques était exacte, au point que si elle fut complétée ensuite sur différents points, elle n'a été sérieusement contestée, sinon dans le fil des années qui suivirent 1968, et qu'aux dépens de la vérité. Ni Soljenitsyne, ni les documents extraits après 1990 des archives soviétiques, n'ont rien appris d'essentiel que ces équipes américaines n'eussent déjà su et exposé. Risquons cette proposition : si l'arme atomique put contenir en partie l'expansion du communisme léniniste mais sans être employée, ce fut à la condition qu'une théorie correcte de ce qu'était ce régime fût à la disposition des dirigeants occidentaux. On peut dire que si le premier programme n'a pas tourné en catastrophe c'est en partie parce que le second a été mené à bien. Du côté américain, la guerre froide a été pilotée les yeux ouverts.

La partie historique proprement dite de ce grand travail s'effectua depuis la chaire de Karpovich. Ce professeur écrivait lui-même fort peu. Il était un Russe de souche, fort attaché à l'Église orthodoxe, pourvu d'un don pédagogique, d'un esprit de vérité et de bon sens qui mit ses élèves sur le bon chemin. Quels élèves ? Je ne cite que ceux que j'ai connus personnellement, Malia, Pipes, Ulam, Treadgold et Marc Raeff. Il y en eut d'autres. Les « élèves de Karpovich » sont une école, et font une légende, comparable à d'autres noyaux de pensée, l'Encyclopédie, le Stift de Tübingen, avec moins d'ambition sublime et une exigence plus sobre et critique de vérité.

La thèse d'habilitation de Marc Raeff porte pour titre : Michael Speransky, et en sous-titre, bien utile à ceux qui n'ont jamais entendu parler de ce personnage : Statesman of Imperial Russia, 1772-1839. L'exemplaire que j'ai entre les mains est paru en Hollande, chez Nijhoff, en 1957. Il se peut que je l'aie lu peu après sa parution, puisque je commençai cette année-là mon apprentissage russe. Ce fut un émerveillement. Je ne l'ai pas relu, mais je crois l'avoir encore en mémoire. C'est de l'histoire philosophique dans la « grande manière ». Y est posé un problème essentiel de la Russie. Comment apporter un ordre rationnel au magma assez primitif et profondément médiéval de l'ancienne Russie, alors que cette rationalisation est déjà 
effectuée dans les Allemagnes et que le Nouveau Régime, consécutif à la Révolution française, est en train de délégitimer même les anciens régimes modernisés, comme celui de la Prusse, le modèle de Speransky? L'exposé de Raeff est lumineux. La clarté et la force du raisonnement sont en soi une leçon pour tout historien, qu'il ait ou non la Russie comme champ d'études. L'échec du projet Speransky est paradigmatique. Jusqu'à sa chute, l'État pétersbourgeois produira des Speransky, lucides, énergiques, intelligents, et tous, sur le point de réussir, échoueront. Raeff montre une grande et savante attention pour le droit, piste fructueuse qu'il ne fera que développer dans la suite de son œuvre et qui est assez rare parmi les historiens de sa génération. Les ouvrages universitaires américains, en général bien présentés, sont tirés à un tout petit nombre d'exemplaires. Je ne trouve pas le Speransky dans les sites de recherche de livres. Il serait souhaitable qu'il soit réimprimé. Et bien sûr traduit en français. Je reconnais que c'est plus utopique encore que le projet de Speransky.

Je ne vais pas analyser les quelque dix ou douze ouvrages de Marc Raeff. Ils figurent en première place dans les bibliothèques des slavisants sérieux. Il a rassemblé ses articles dans un recueil, Political Ideas and Institutions in Imperial Russia. On y trouvera l'essentiel de sa pensée, laquelle s'approfondit sans changer parce qu'il avait dès le départ une vision juste du pays. The Well Ordered Police State (1983) est un maître livre d'histoire comparée. Mettant à profit sa culture juridique, Marc Raeff montre comment la constitution d'un ordre administratif et légal a transformé l'Europe occidentale et principalement l'Allemagne, en l'absence d'un État monarchique centralisé. Comme le résume l'historien Orest Ranum : «l'argument est fondamental : l'administration étatiste dans l'Europe de l'Ouest sortait des institutions locales, rationalisées par le caméralisme ; à l'Est, ce même type d'étatisme ne pouvait qu'être imposé, sur une société encore seigneuriale, avec un résultat beaucoup plus brutal, vite perçu comme non russe ». C'est ainsi que l'imposition forcée des normes européennes au lieu de moderniser fait reculer dans l'archaïsme1. Il me faut signaler une anthologie, préfacée par Isaiah Berlin qui m'a accompagné depuis sa parution : Russian Intellectual History, qui offre le panorama le plus complet de la pensée russe, depuis Feofan Prokopovich jusqu'à Ivanov et Gershenzon. Quand Malia produisit dans notre langue son Comprendre la Révolution russe, je proposai à Marc Raeff d'écrire le livre complémentaire, ce qu'il fit, directement en français : Comprendre l'Ancien régime russe. Après ces deux livres, si on n'a pas compris, c'est à désespérer. Nous parlions souvent avec la même admiration du chef-d'œuvre d'Anatole Leroy-Beaulieu, L'Empire des tsars et les Russes. J'arrachai à la direction de la collection Bouquins la décision de le republier, mais à la condition que Marc Raeff en rédige la préface. Ample et riche préface, qui situe ce grand esprit, aujourd'hui négligé, à la place exacte entre Tocqueville, avec qui il voulait rivaliser en écrivant sur la Russie le symétrique de

1. Phénomène que Martin Malia a un peu méconnu quand il remarque que les institutions russes n'avaient, au XIX ${ }^{\mathrm{e}}$ siècle que quelques décennies de retard sur l'évolution des institutions allemandes. L'Allemagne adaptait les nouvelles institutions à une société prête à les faire fructifier. Le placage des mêmes institutions sur la société russe avait parfois un effet contraire et duricissait les résistances à la modernisation. 
La Démocratie en Amérique, et les penseurs plus positivistes de la fin du siècle, Renan et Taine. Cette republication ne fit pas la fortune de l'éditeur.

En 1961, Marc Raeff, quitta Clark University, son premier poste, et il fut élu à Columbia puis nommé Bekhmeteff Professor of Russian Studies. Il n'en bougea plus. C'est dans cette université que je fis sa connaissance et je voudrais donner ici un témoignage plus personnel.

C'était en 1962. Marc Raeff, sur invitation de Portal, professeur à la Sorbonne, prit à Paris une année sabbatique, se résignant avec sa femme et sa petite fille à des conditions de logement fort médiocres et prouvant ainsi une affection méritoire et tenace pour notre capitale. Nous devînmes amis, c'est-à-dire qu'il consentit à converser longuement avec le jeune homme fort ignorant que j'étais. Je venais de passer une année en URSS, expérience décisive que je mis des années, grâce en particulier à Raeff, à analyser convenablement. Il m'invita à Columbia, sur une bourse Ford et je passai à New York l'année 1964-1965. J'étais fort intimidé. Nous étions encore dans ce magnifique apogée de l'Amérique, qui durait depuis la guerre et qui allait se flétrir quelque peu dans les années suivantes. Le Russian Center de Columbia fonctionnait comme une montre. Les séminaires étaient dirigés par des personnalités considérables qui me faisaient, sans le vouloir, découvrir mon incompétence. Je dois dire qu'il eût été difficile en France d'acquérir une meilleure connaissance. Je suivis, en particulier quelques séminaires de Brzezinski qui me fit, à juste titre, grande impression, mais plus régulièrement les cours et les séminaires de Raeff. Je m'imprégnai de son enseignement, dispensé avec cette simplicité et cette modestie qui le caractérisaient, mais souvent, comme il voyait que je m'ennuyais un peu tout seul dans l'hiver new yorkais, il acceptait de faire de longues promenades et d'ouvrir devant moi les trésors de sa sagesse intellectuelle, qui s'étendait bien au-delà des affaires de la Russie. Je devenais décidément son élève.

Depuis nos relations sont devenues celles de vieux amis. Nous échangions nos enfants pendant les vacances. À Paris, il descendait dans un hôtel à quelques pas de ma maison. De cette amitié, il reste une trace écrite : les deux épais cartons où j'ai conservé ses lettres. Elles sont écrites à la main, sauf les toutes dernières quand il ne pouvait plus manier le stylo. J'imagine que ces lettres, écrites sur quarante-cinq ans par un très distingué professeur pourront un jour intéresser quelqu'un. Elles sont vraiment intéressantes.

J'ai dit que le climat intellectuel aux États-Unis, vers 1965, était entré dans l'ère des troubles dont il n'est pas vraiment sorti. Dans le domaine des études russes, les résultats devenus presque canoniques de l'école de Harvard - étendue par la dispersion des élèves de Karpovich sur les deux côtes Est et Ouest - commencèrent à être radicalement contestés ${ }^{2}$. Cette contestation, Malia, une des victimes, l'attribuait au « Acquired Immunodeficient Menshevik Syndrom ». Je ne veux pas entrer dans cette bataille, sinon pour dire que les anciens ne capitulèrent en rien et

2. Un bon symbole de ce tournant est le changement de titre du classique de Merle Fainsod, dans une réédition posthume : au lieu de How USSr is ruled: How USSR is governed. 
qu'ils eurent le temps, s'ils vivaient encore, d'être justifiés par les élèves des contestataires, lesquels reconnurent que c'était eux et non leurs nouveaux maîtres qui avaient dit la vérité.

Raeff fut la cible d'attaques tortueuses de certains collègues de Columbia. D'humeur pacifique, il dédaigna de répondre et, aussitôt qu'il put, il prit sa retraite et se retira dans le sein des doctes muses.

Il continua de lire, d'écrire, de fréquenter avec mesure quelques colloques, de donner quelques leçons en Allemagne et en France, de faire quelques voyages de plaisir et de gourmandise en Italie et en France. Avec nos Cahiers du Monde russe et soviétique, il collabora fidèlement. Il ne changeait pas, pareil à soixante-quinze ans qu'à quarante. Il me dit à la fin de l'année dernière qu'il était frappé de sclérose latérale amyotrophique, dite aussi maladie de Charcot, mortelle à coup sûr. Il s'éteignit, semblable à lui-même, bon, amène, cordial, reconnaissant, serein, chéri par sa femme et ses deux filles.

Je voudrais définir, en comparaison d'autres « élèves de Karpovich » quel était le style distinctif de Raeff comme historien de la Russie. Il est possible, et il était le premier à le reconnaître, que Malia a plus d'éclat intellectuel et Pipes plus de puissance $^{3}$. On peut l'admettre. Mais Raeff, dont la culture était certainement égale, avait une vertu discrète et pénétrante : tout ce qu'il affirmait était vrai, purement et simplement vrai. Sur la Russie en général, j'ai eu quelques différents avec Malia, je n'ai pas toujours été d'accord avec Pipes, mais je me reposais complètement et tranquillement sur Raeff qui ne se trompait jamais. Dans les vingt dernières années, il n'a commis sur la Russie aucune erreur. Cette infaillible boussole ne nous guidera plus .

Alain Besançon

3. Raeff trouva le moyen d'être l'ami de ces deux ennemis irréconciliables. 